\title{
A Retrospective Review of Facial Fractures in Wales
}

\author{
Jayan George ${ }^{1, *} \mathbb{C}$, Pranter Brahmabhatt ${ }^{2}$, Amir Farboud ${ }^{2}$ and Conor Marnane ${ }^{2}$
}

1 General Surgical Department, Sheffield Teaching Hospitals NHS Foundation Trust, Herries Road, Sheffield S5 7AU, UK

2 ENT Head and Neck surgery department, Morrison Hospital, Swansea SA6 6NL, UK; pranter_b@hotmail.com (P.B.); amirfarboud@doctors.net.uk (A.F.); conor.marnane@wales.nhs.uk (C.M.)

* Correspondence: jayan.george@aol.com Tel.: +44-1142-434-343; Fax: +44-1142-266-986

Received: 17 October 2018; Accepted: 15 November 2018; Published: 19 November 2018

\begin{abstract}
Facial fractures are the third most common type of fracture, with the nose most frequently involved. In the United Kingdom, their incidence is rising among young males particularly, and recent reports suggest that nasal injuries in females are becoming more common, which is attributed to "ladette" culture. The aim of this study was to investigate if there is any correlation in Wales with the rise in female facial fractures reported in England. A retrospective analysis of National Health Service Wales Informatics Service data (NHS WIS) shows that facial fractures were more likely to occur in males, in their teens or twenties, most commonly caused by assault and linked to alcohol consumption in this demographic. The most common cause of facial fracture in Wales was due to an accident which differs from England. The overall number of facial fractures in both males and females did not change significantly. It may be that the binge drinking culture is not as prevalent in Wales as it is in England. The reasons for this may be mutifactorial, reflecting geographical, cultural and socio-economic factors. Our findings do not correlate with the literature regarding the epidemiology of facial fractures. We suggest that binge drinking and "ladette culture" is not seen as commonly in Wales from the point of view of the ill effects of alcohol consumption.
\end{abstract}

Keywords: facial injuries; nasal bones; social behaviour; Wales

\section{Introduction}

In the United Kingdom (UK), facial fractures are the third most common type of fracture, with the nose most frequently involved [1]. Recent reports suggest that the incidence of nasal fractures is increasing, particularly in females, most likely due a rise in "ladette" culture [2]. The majority of facial fractures in Europe are caused by assault or road traffic collisions (RTC) [3,4]. Up-to-date published data are sparse regarding exact figures of England and Wales, a recent epidemiological review identified three England based papers and found assault to be the most common cause [3,5-7]. Other significant causes include falls and sports injuries [8,9]. Nasal fractures are also the most common type of facial fracture in children $[10,11]$. We aim to review the pattern and epidemiology of facial fractures in Wales to ascertain if they correlate with recent reports from England.

\section{Materials and Methods}

Retrospective data was obtained from the National Health Service Wales Informatics Service (NHS WIS) for admissions in Wales from 2000 to 2013. All patients who had a principal diagnosis of a facial fracture or nasal fracture and an associated operation were included. Data was also obtained from the NHS WIS for all accident and emergency department (A\&E) attendances for facial injury from 2009 to 2013. Analysis of data was performed using IBM SPSS Statistics 20. No ethical approval was required for this study. 


\section{Results}

From 2000 to 2013, a total of 17,547 operations were performed for all types of facial fractures in all the health boards across Wales; 14,694 (83.7\%) were for males and 2853 (16.3\%) were for females (Figure 1). During this period, the greatest number of operations performed for facial fractures was in 2009 (1498 operations). The 20-29 age group comprised the highest number of operations for facial fractures with 6574 patients of this age group treated (37.5\% of total admissions). Of the various operations for facial fractures, 9161 (52.2\%) were on the nasal bones (Figure 2). Statistical analysis using the Pearson rank correlation shows that there is no significant change $(p>0.05)$ in the numbers of facial injuries in either males or females over the 13 years.

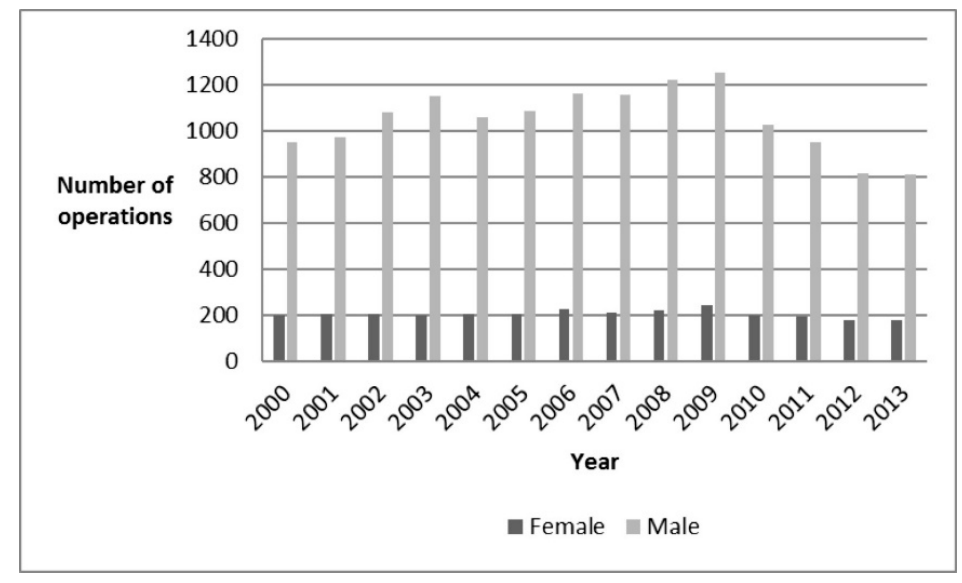

Figure 1. The number of operations performed per year on males and females for all types of facial fractures across all the health boards in Wales for the period 2000-2013.

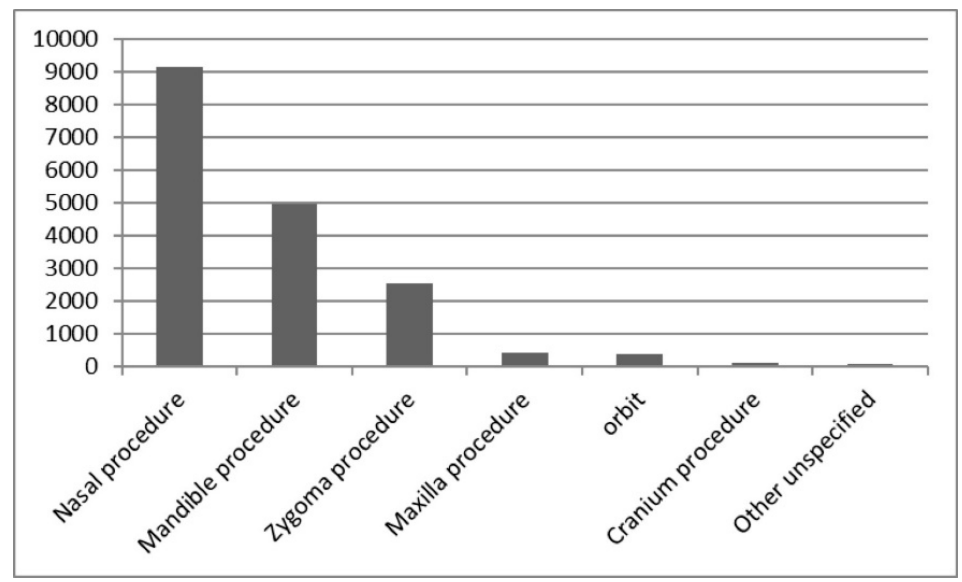

Figure 2. The number of operations for facial fractures performed across Wales categorised by location on the face.

From 2009 to 2013 there were 3669 A\&E admissions for facial injuries, 2380 (64.9\%) of these were due to an accident (including RTC), 904 (24.6\%) due to assault and the remaining cases were unspecified (Figure 3). The proportion of fractures caused by assault, $776(85.8 \%)$ occurred in men, and $128(15.2 \%)$ occurred in women (Figure 4$)$. The involvement of alcohol was reported in $221(6 \%)$ of attendances, in 2075 (56.6\%) attendances it was denied and, in the remainder, no association was documented. The 20-29 age group represented the largest proportion of attendances for facial fractures involving alcohol consumption and caused by assault compared to any other age group (Figure 5). Older patients more commonly attended with a facial fracture due to a fall (Figure 6). Attendances involving road traffic collisions (RTCs) and sports were more common in males than in females, shown in Figures 7 and 8, respectively. 


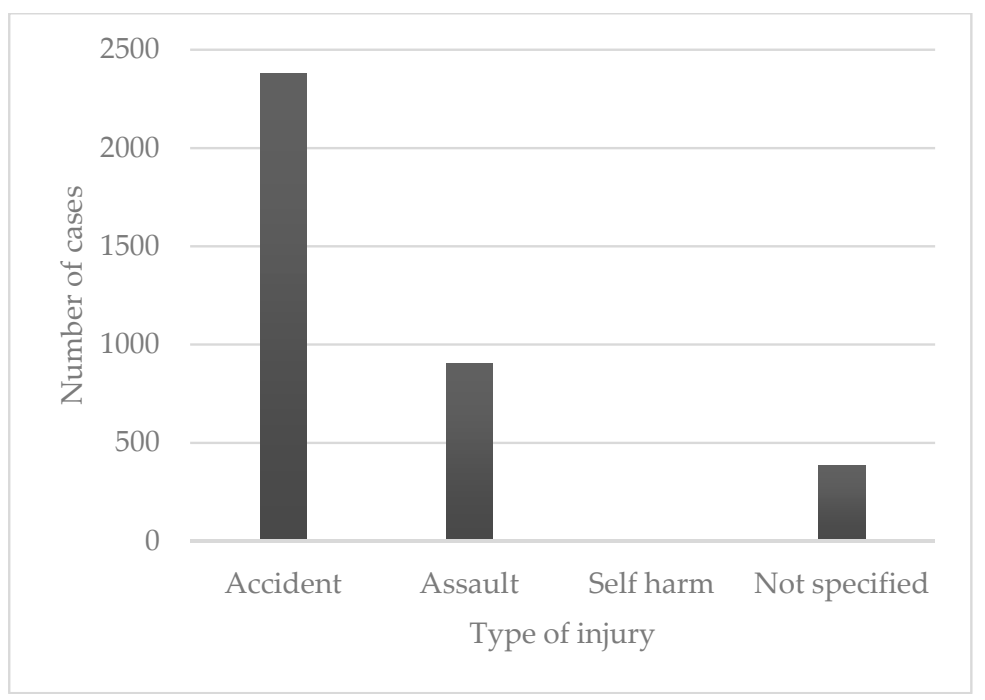

Figure 3. All accident and emergency department (A\&E) admissions for facial injury between 2009-2013 showing the proportions caused by accident and those caused by assault.

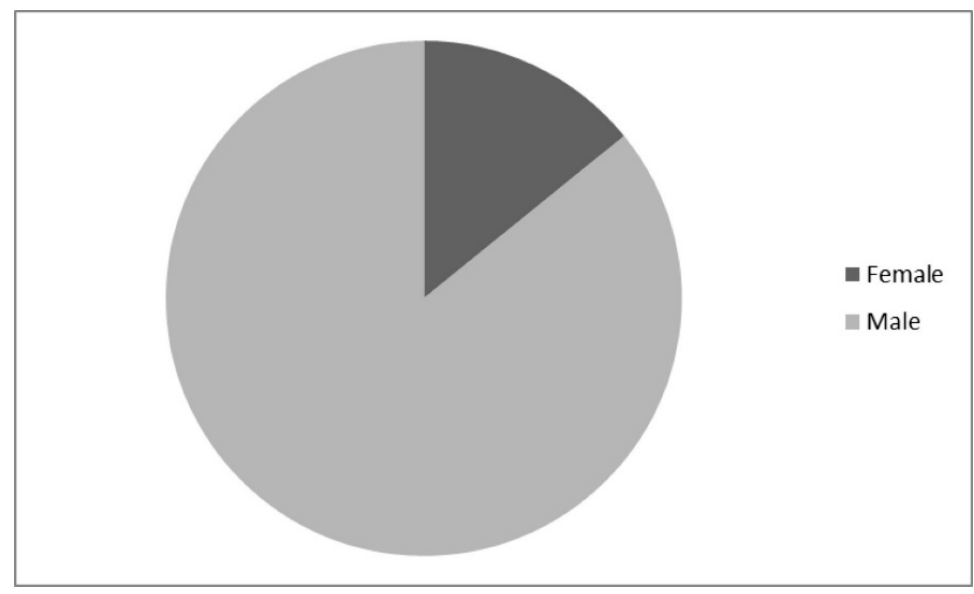

Figure 4. All fractures caused by assault are shown according to the proportion caused in men and those caused in women.

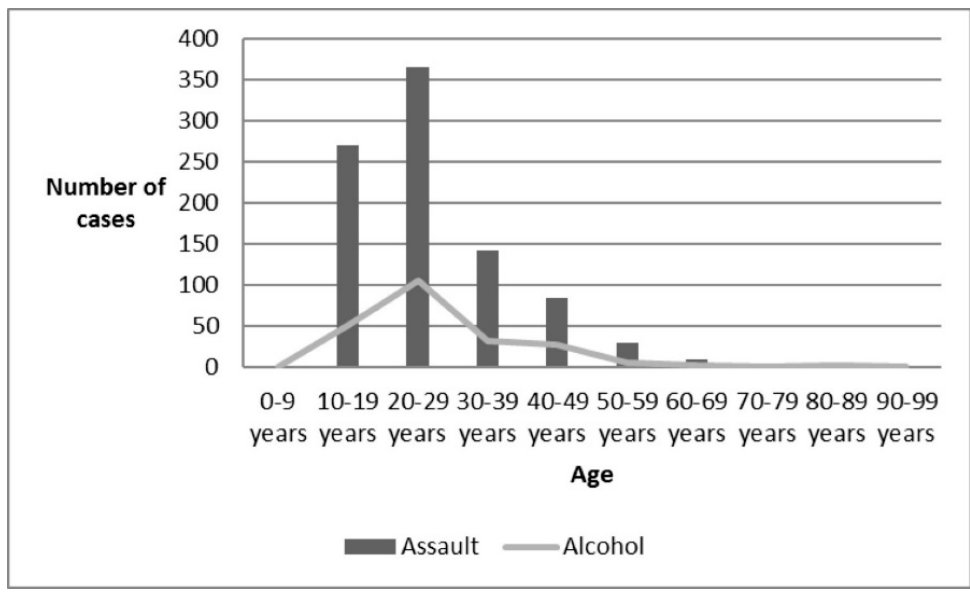

Figure 5. The incidence of facial fractures caused by assault at each decade of age and the involvement of alcohol in each case. 


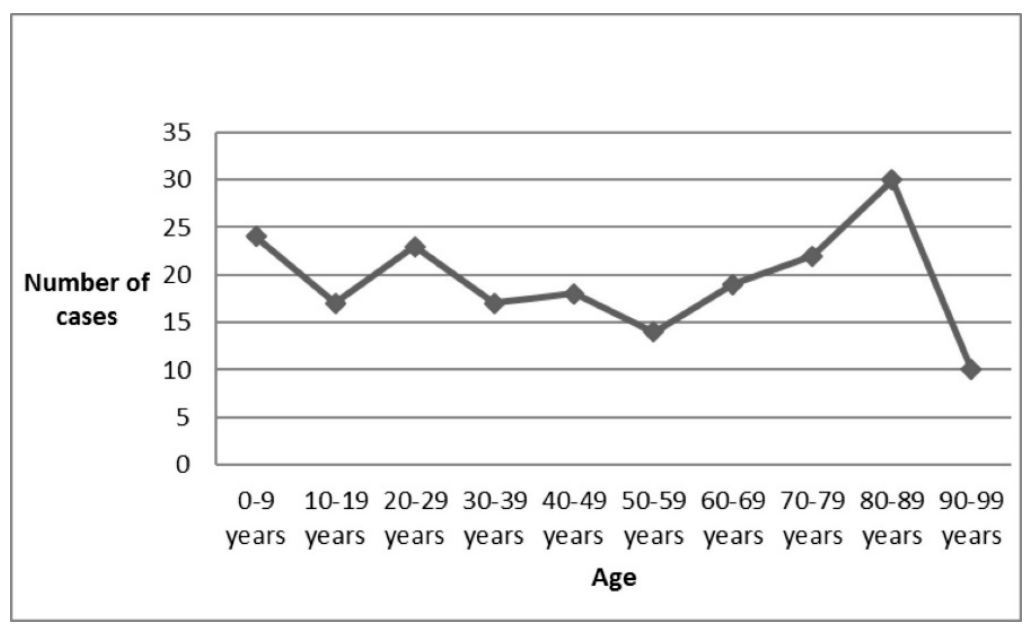

Figure 6. The incidence of a fall being reported as the cause of the facial fracture at each decade of age.

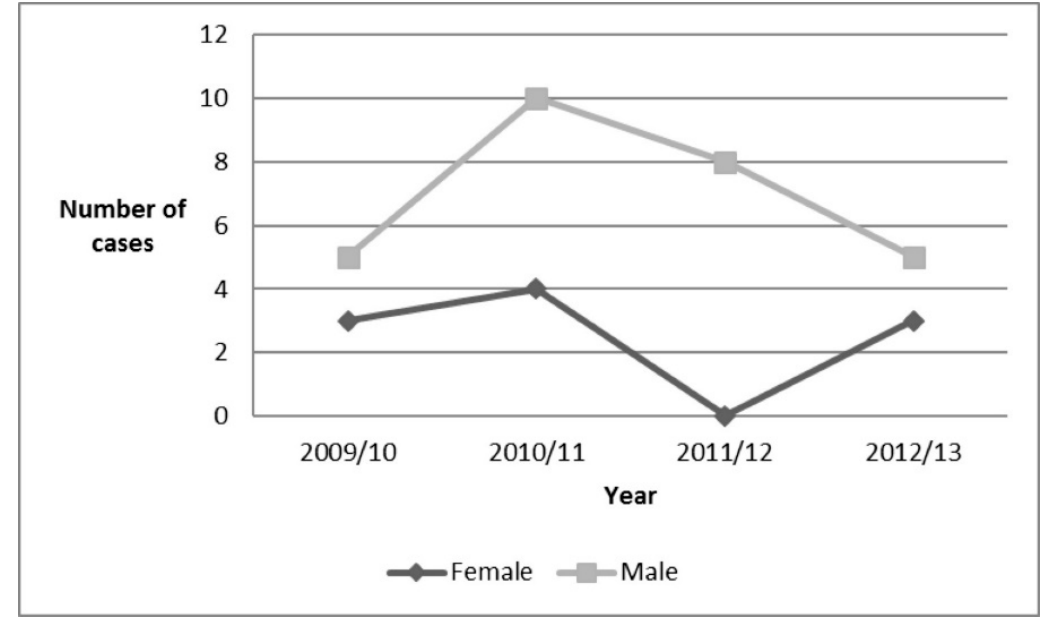

Figure 7. The incidence of a road traffic collision being the cause of the facial fracture in both males and females per year from 2009-2013.

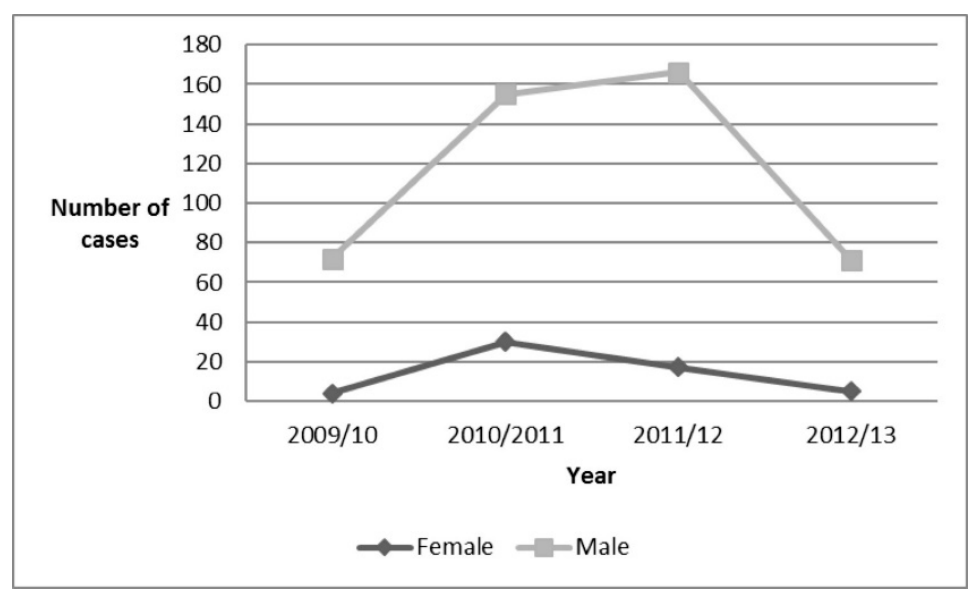

Figure 8. The incidence of a sports related injury being the cause of the facial fracture in both males and females per year from 2009-2013.

\section{Discussion}

This study uses two different data sources to answer the question whether incidence of facial fractures are increasing. Data from NHS WIS for admissions from 2000 to 2013 provide the incidence 
of facial and nasal fractures across Wales whereas data from A\&E attendances for facial injury from 2009 to 2013 reveal the nature of these injuries.

Our results show that the most common cause of facial fractures in Wales is due to an accident, as opposed to previous data published from the UK (which focuses mainly on data from England) [3]. The most common cause of facial fractures worldwide is RTC, especially in the non-western world, which could be due to the more stringent laws of wearing seat belts [3]. The increasing elderly population in Wales could also be responsible for this, as falls are more common in the elderly population.

Our results seem to indicate a decline in the number of males with facial fractures, particularly in the period 2010 to 2013. However, statistically, this was not significant in the numbers of this injury in either males or females over the 13 years. Of these injuries, the nose is the most common site involved, which is in keeping with the literature [1,2].

We found that the 20-29 age group had the greatest number of facial fractures caused by assault and involving alcohol. This correlates well with the findings of Hutchison et al. who discovered 15-25-year-olds had the highest A\&E attendances for assault involving alcohol [12]. The same study found that men had more facial injuries due to assault than women, in a ratio of 4:1. Our results found this ratio to be $5: 1$.

Previous studies have suggested that over half of facial injuries due to assault involved alcohol consumption [13]. Our data suggests about $15 \%$ of cases due to assault involved alcohol. As our data rely on the information that was given during the A\&E admission, it is possible that some information was withheld by the patient, the documentation by the admitting doctor was inaccurate or subsequent coding was incomplete, leading to the above discrepancy.

Of those attendances due to an accident (defined as a slip, trip or fall), there were bimodal peaks in incidence in the young and in the elderly, which corresponds well with the current literature $[14,15]$.

The fact that more operations are performed on elderly females with facial fractures may be due to several factors including cosmetic appearance and greater incidence of osteoporosis among women. Furthermore, as women, on average, live longer than men in the senior age group, it is unsurprising that more women sustained fractures than men. The most recent figures released by the Welsh Government states that, on average, women live to 82.2 years compared to men who, on average, live to 78.2 years [16].

Sports injuries were shown to be more common in males than females, which also correlates well with the current literature [17]. A similar result was seen in facial fractures caused by RTC. In both these categories there was a large variation on a year-by-year basis but little change in the overall trend across the dataset.

Our results show that the incidence of facial fractures has not changed over the course of the 13 years included in this study and is not in keeping with the data from England. It is likely that binge drinking and "ladette" culture has not seen a similar increase in Wales as it has in England, therefore, not producing the same upward trend in facial fracture numbers evident in the data from England. The reasons for this are most likely multifactorial but may be partly attributed to lower incomes in Wales compared to England; the Office for National Statistics reports a gross disposable household income per head of $£ 17,066$ in England and $£ 14,623$ in Wales [18]. There are potential geographical factors which might explain the difference in our data to that from England. A greater separation between the population and healthcare services is more likely in Wales than in England. For these reasons, patients with minor nasal injuries may not seek medical attention and therefore would not feature in our results.

Our findings largely correlate with the literature regarding the epidemiology of facial fractures. Facial fractures are more common in males and in people in their teen years or twenties. Injuries associated with assault, alcohol consumption, sports and road traffic collisions are all more common in males. Accidental injuries are more common in the elderly population, with elderly females undergoing more operations for facial fractures than males of the same age group. 
Across Wales, we found no change in the numbers of facial fractures in both males and females, which does not entirely correlate with the data from England. While there is an expected and demonstrated link between alcohol consumption and increased numbers of facial fractures, our results demonstrate no change in the incidence of facial fractures across the 13-year period we studied. It is likely that "ladette culture" is not as prevalent in Wales as it is in England. One factor contributing to this could be an average lower disposable income in Wales. There may be other reasons for these findings, such as geography and differences in health-seeking behaviour. Nevertheless, increased alcohol consumption continues to result in ill-effects and this trend in anti-social behaviour needs to be addressed by way of minimal pricing for alcohol, by improved government policy towards binge drinking, by focusing on attitudes towards drinking, through advertising, and a targeted health promotion. We feel our retrospective review supports the introduction of such legislation and patient education. Is it possible that austerity has played a part in this decline, and that because of the lack of disposable income and a decline in binge drinking, we are seeing a trend in behaviour, which may have implications for policy makers.

\section{Conclusions}

In the UK, there has been a recent increase in nasal injuries particularly amongst young women; attributed by some studies to a rise in "ladette culture". This study investigates whether this rise is across the whole of the UK. Overall, the incidence of facial fractures did not change significantly in Wales over the 13-year period included in this study. This would suggest the rise in facial fractures is represented more in the data from England. The most common cause of facial fracture in Wales is due to accident. Facial fractures are more common in males and in people in their teen years or twenties. Injuries associated with assault, alcohol consumption, sports and road traffic collisions are all more common in males. Accidental injuries are more common in the elderly, with females undergoing more operations for facial fractures than males of the same age group. "Ladette culture" is not as prevalent in Wales as it is in England. The cause for this is most likely multifactorial; lower incomes, differences in health-seeking behaviour and geography. Our findings advocate and support government legislation to increase health promotion and limit the ill effects of alcohol consumption, particularly anti-social behaviour.

Author Contributions: J.G.: Developing and proofing the manuscript and editing the images. P.B.: Developing, statistical analysis and proofing the manuscript. A.F.: Developing and proofing the manuscript. C.M.: Developing and proofing the manuscript.

Funding: This research received no external funding.

Acknowledgments: The authors would like to thank Samuel Birks for editing the images. We would like to thank Susan Prosser, Librarian at Singleton Hospital, for her assistance with the literature review. We also wish to thank Lesley Stroud for her assistance with obtaining data from theatre data using the Theasis database.

Conflicts of Interest: The authors declare no conflict of interest.

\section{References}

1. Renner, G.J. Management of nasal fractures. Otolaryngol. Clin. N. Am. 1991, 24, 195-213.

2. Trinidade, A.; Buchanan, M.A.; Farboud, A.; Andreou, Z.; Ewart, S.; Mochloulis, G.; Kothari, P.; Frosh, A.C.; Vlastarakos, P.V. Is there a change in the epidemiology of nasal fractures in females in the UK? J. Laryngol. Otol. 2013, 127, 1084-1087. [CrossRef] [PubMed]

3. Boffano, P.; Kommers, S.C.; Karagozoglu, K.H.; Forouzanfar, T. Aetiology of maxillofacial fractures: A review of published studies during the last 30 years. Br. J. Oral Maxillofac. Surg. 2014, 52, 901-906. [CrossRef] [PubMed]

4. Jaberoo, M.-C.; Joseph, J.; Korgaonkar, G.; Mylvaganam, K.; Adams, B.; Keene, M. Medico-legal and ethical aspects of nasal fractures secondary to assault: Do we owe a duty of care to advise patients to have a facial X-ray? J. Med. Ethics 2013, 39, 125-126. [CrossRef] [PubMed] 
5. Rashid, A.; Eyeson, J.; Haider, D.; van Gijn, D.; Fan, K. Incidence and patterns of mandibular fractures during a 5-year period in a London teaching hospital. Br. J. Oral. Maxillofac. Surg. 2013, 51, 794-798. [CrossRef] [PubMed]

6. Hill, C.M.; Crosher, R.F.; Carroll, M.J.; Mason, D.A. Facial fractures-The results of a prospective four-year-study. J. Maxillofac. Surg. 1984, 12, 267-270. [CrossRef]

7. Timoney, N.; Saiveau, M.; Pinsolle, J.; Shepherd, J. A comparative study of maxillo-facial trauma in Bristol and Bordeaux. J. Craniomaxillofac. Surg. 1990, 18, 154-157. [CrossRef]

8. Baring, D.E.C.; Bowyer, D.J.; Adamson, R. Patient Self-assessment of Nasal Fractures and Self-referral to an Ear, Nose, and Throat Department. Otolaryngol. Neck Surg. 2012, 146, 913-917. [CrossRef] [PubMed]

9. Baring, D.; Murray, C.; Singh, J.; Davidson, A.; Syed, M.I. Prospective, blinded study of nasal injuries: Comparison of doctor and nurse assessment. J. Laryngol. Otol. 2009, 123, 1338-1342. [CrossRef] [PubMed]

10. Kidd, A.J.; Beattie, T.F.; Campbell-Hewson, G. Facial injury patterns in a UK paediatric population aged under 13 years. Emerg. Med. J. 2010, 27, 603-606. [CrossRef] [PubMed]

11. Anderson, P.J. Fractures of the facial skeleton in children. Injury 1995, 26, 47-50. [CrossRef]

12. Hutchison, I.L.; Magennis, P.; Shepherd, J.P.; Brown, A.E. The BAOMS United Kingdom survey of facial injuries Part 1: Aetiology and the association with alcohol consumption. British Association of Oral and Maxillofacial Surgeons. Br. J. Oral Maxillofac. Surg. 1998, 36, 3-13. [CrossRef]

13. Gerber, B.; Ahmad, N.; Parmar, S. Trends in maxillofacial injuries in women, 2000-2004. Br. J. Oral Maxillofac. Surg. 2009, 47, 374-377. [CrossRef] [PubMed]

14. Rehman, K.; Edmondson, H. The causes and consequences of maxillofacial injuries in elderly people. Gerodontology 2002, 19, 60-64. [CrossRef] [PubMed]

15. Shaikh, Z.S.; Worrall, S.F. Epidemiology of facial trauma in a sample of patients aged 1-18 years. Injury 2002, 33, 669-671. [CrossRef]

16. Welsh Government. Life Expectancy by Gender and Year. Stats Wales 2012. Available online: https:/ / statswales.gov.wales/Catalogue/Health-and-Social-Care/Life-Expectancy/LifeExpectancyby-Gender-Year (accessed on 23 March 2017).

17. Hill, C.M.; Burford, K.; Thomas, D.W.; Martin, A. A one-year review of maxillofacial sports injuries treated at an accident and emergency department. Br. J. Oral Maxillofac. Surg. 1998, 36, 44-47. [CrossRef]

18. Office of National Statistics. Compendium of UK Statistics. 2014. Available online: http:/ / webarchive.nationalarchives.gov.uk/20160105160709/http:/ /www.ons.gov.uk/ons/guidemethod/compendiums/compendium-of-uk-statistics/economy/index.html (accessed on 23 March 2017). 